\title{
Rapid identification of bioactive carbohydrazide reaction products by an LC-DAD-SPE-NMR approach
}

\author{
Iva Habinovec, Tomislav Jednačak and Predrag Novak* \\ University of Zagreb, Faculty of Science, Department of Chemistry, Horvatovac 102a, 10000 Zagreb, Croatia \\ ihabinovec@chem.pmf.hr; tjednacak@chem.pmf.hr
}

*Corresponding Author: E-mail: pnovak@chem.pmf.hr; Tel.: +385-1-460-6184; Fax: +385-1-460-6181

Received: November 09, 2015; Revised: December 24, 2015; Published: December 30, 2015

\begin{abstract}
On-line coupling of high performance liquid chromatography with diode array detection to solid phase extraction combined with nuclear magnetic resonance (LC-DAD-SPE-NMR) was used to monitor carbohydrazide condensation reaction progress. First, a chromatographic method was developed and optimised and individual peak separation was readily achieved by using an isocratic acetonitrile-phosphate buffer mobile phase. Subsequently, separated compounds were trapped on SPE cartridges and dried with nitrogen gas. Peak elution was then performed with deuterated acetonitrile and sent for NMR analysis. Single and multiple trapping options were applied. One-and two-dimensional NMR spectra were recorded using a Prodigy cryoprobe. It was demonstrated that LC-DAD-SPE-NMR setup was proved very useful for rapid and unambiguous identification of the reaction products and for determination of their structure. By using Prodigy cryoprobe in NMR measurements we were able to detect and identify compounds present at microgram level thus proving a high sensitivity of this methodology for monitoring reactions of bioactive molecules and drugs.
\end{abstract}

\section{Keywords}

carbohydrazide derivatives; reaction monitoring; LC-DAD-SPE-NMR; product identification; structure elucidation

\section{Introduction}

Aromatic imines derived from carbohydrazide are promising therapeutic agents with a broad spectrum of biological properties, such as antiviral, antitubercular, antifungal, cytotoxic and antimalarial activity [14]. Furthermore, they were reported to be used as precursors in the synthesis of various nitrogen and sulphur containing heterocycles $[5,6]$. Owing to their ability to act as chelate ligands and stabilise metal ions in different oxidation states, carbohydrazide Schiff bases have also found applications as multitopic ligands for the targeted construction of bioactive coordination systems [7,8]. These compounds can easily be prepared by the condensation reaction between carbohydrazide and appropriate aldehyde or ketone. The condensation of carbohydrazide (I) with salicylaldehyde (II) yielded a mixture of mono- (III) and bis(salicylidene)carbohydrazide (IV), as shown in Figure $1[9,10]$. 


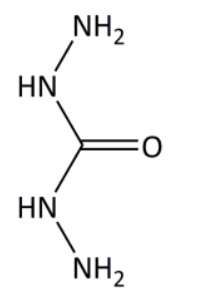

I<smiles>O=Cc1ccccc1O</smiles>

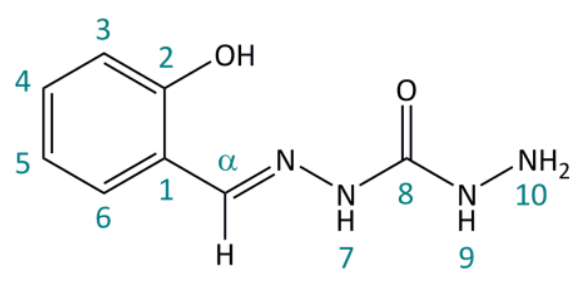

III<smiles>C1CCC1</smiles><smiles>O=C(N/N=C/c1ccccc1O)N/N=C/c1ccccc1O</smiles>

IV

Figure 1. The condensation reaction between carbohydrazide and salicylaldehyde.

We have recently reported that in-line Raman spectroscopy combined with multivariate data analysis could be applied for in-line monitoring of condensation reaction and for assessing the mixture content [11]. However, several drawbacks of this methodology, such as low sensitivity, complexity of the reaction mixture, and severe band overlapping, prevented precise identification of the compounds in the reaction mixture and determination of the reaction endpoints directly from the in-line spectra. In order to resolve these issues, we employed here an approach based on the hyphenated system comprising of high performance liquid chromatography with diode array detection (LC-DAD), solid phase extraction (SPE) and nuclear magnetic resonance spectroscopy equipped with a cryogenically cooled probe (cryo NMR). This LCDAD-SPE-NMR setup represents a state-of-the-art technology for rapid and accurate mixture analysis. The coupling of HPLC with NMR spectroscopy has become one of the most efficient and powerful analytical technique to analyse complex mixtures. Identification and structural characterization of compounds in a mixture without their physical separation has long been a dream of analytical chemists. LC-NMR hyphenation gives a wealth of structural information on small quantities of substances without the necessity of their isolation and purification [12-16]. A further coupling of LC-NMR to SPE has considerably improved the sensitivity of this technique by post column peak trapping onto the SPE cartridges enabling the separate compounds to be concentrated and eluted fully with deuterated solvents. LC-SPE-NMR is now widely accepted tool for dereplication and discovery of natural products from complex mixtures of plants, metabolites, drug impurity profiling and environmental analysis [17-21]. On the other hand, it has been less frequently used to monitor chemical reaction products $[22,23]$. Hence, in the present work we applied this hyphenated method to analyse carbohydrazide condensation reaction. One- and two-dimensional NMR experiments with a NOESY-type double presaturation for suppressing acetonitirile and water resonances will be used for structure elucidation of mixture components.

\section{Experimental}

\section{Chemicals}

Salicylaldehyde (min. $98 \%$ ) and carbohydrazide (min. $98 \%$ ) were purchased from Sigma-Aldrich (MO, USA). Piperidine (min. $99 \%$ ) was purchased from Acros Organics (NJ, USA). Methanol (min. $99.95 \%$ ) was obtained from Carl Roth GmbH (Karlsruhe, Germany). Acetonitrile (HPLC grade) was purchased from J. T. Baker (PA, USA) and potassium dihydrogen phosphate (for chromatography) was obtained from Kemika 
(Zagreb, Croatia). Acetonitrile-D3 (D, $99.8 \%$ ) was purchased from Cambridge Isotope Laboratories, Inc. (MA, USA).

\section{Synthesis}

Carbohydrazide $(0.60 \mathrm{~g}, 6.67 \mathrm{mmol})$ was gradually dissolved in $40 \mathrm{~mL}$ of methanol. After the addition of salicylaldehyde $(0.71 \mathrm{~mL}, 6.67 \mathrm{mmol})$ and a few drops of piperidine, the reaction mixture was heated at 55 ${ }^{\circ} \mathrm{C}$ and stirred under reflux for $40 \mathrm{~min}$ [9]. During heating, a white crystalline product started to precipitate. The product was filtered off, washed with a small amount of cold methanol and dried in air.

\section{LC-SPE analysis}

An Agilent 1260 Infinity HPLC system equipped with quaternary pump, an autosampler, thermostatted column compartment and DAD detector was used for chromatographic separation prior to post-column solid-phase extraction. Separation was performed on an Agilent Poroshell 120 C18 column ( $250 \mathrm{~mm} \times 4.6$ $\mathrm{mm}$ ) with $4 \mu \mathrm{m}$ particle size in analytical mode. Injection volume was $25 \mu \mathrm{L}$ and the flow rate was $1.0 \mathrm{~mL} \mathrm{~min}{ }^{-1}$. Elution was isocratic using a mobile phase consisting of $50 \%$ acetonitrile and $50 \%$ phosphate buffer ( $\mathrm{pH} \mathrm{7)}$ at $298 \mathrm{~K}$ over $10 \mathrm{~min}$. The automatic post-column SPE was performed on a Prospekt 2 LCSPE-NMR interface (Spark Holland, Emmen, The Netherlands). Both single and multi-trapping methods were used for the SPE pre-concentration. Compounds from reaction mixture were trapped on Hysphere Resin GP, C18 and C8 SPE cartridges $(10 \times 2 \mathrm{~mm})$. To increase the retention of the compounds on SPE cartridges a post-column water addition was performed with a flow rate of $3.0 \mathrm{~mL} \mathrm{~min}^{-1}$ using the Knauer K-120 HPLC pump (Knauer, Berlin, Germany). After the cartridges were dried with nitrogen gas for 59 min, compounds were eluted with $336 \mu \mathrm{L}$ of deuterated acetonitrile into the $3 \mathrm{~mm}$ NMR tubes.

\section{NMR spectroscopy}

NMR spectra with NOESY-type solvent suppression module were recorded on Bruker Avance III HD 400 spectrometer operating at $400 \mathrm{MHz}$ equipped with a broadband observed (BBO) $5 \mathrm{~mm}$ Prodigy cryoprobe and z-gradient accessories. After LC separation and peak trapping on the SPE unit, the separated components were dissolved in acetonitrile- $d_{3}$ and measured in $3 \mathrm{~mm}$ tubes with MATCH inserts for adapting the tubes to $5 \mathrm{~mm}$ Bruker spinners. All experiments were carried out at $298 \mathrm{~K}$ using TMS as an internal standard.

Proton spectra with spectral width of $6002 \mathrm{~Hz}$ and a digital resolution of $0.36 \mathrm{~Hz}$ per point were measured with 64 and 128 scans. In the gCOSY experiment, 2048 points in the $f 2$ dimension and 128 increments in the $f 1$ dimension were used. For each increment, 16 scans and the spectral width of $6010 \mathrm{~Hz}$ were applied. Digital resolution was 5.87 and $93.78 \mathrm{~Hz}$ per point in $f 2$ and $f 1$ dimensions, respectively.

The gHSQC and gHMBC spectra were acquired with 64 and 128 scans, respectively. Spectral width was $6009 \mathrm{~Hz}$ in $f 2$ and $20124 \mathrm{~Hz}$ in $f 1$ dimension for both experiments. $1 \mathrm{~K}$ data points were applied in the time domains and for each data set 256 increments were collected. The resulting digital resolution was $5.86 \mathrm{~Hz}$ per point in $f 2$ dimension and $314.4 \mathrm{~Hz}$ per point in $f 1$ dimension.

\section{Results and Discussion}

The condensation reaction of carbohydrazide and salicylaldehyde was performed in methanol and the reaction products were subsequently analysed by LC-DAD-SPE-NMR method. Prior to LC-DAD-SPE-NMR analysis, a chromatographic method was developed and optimised. Chromatographic separation of the individual products and reactants was readily achieved by using an isocratic acetonitrile-phosphate buffer 
mobile phase. To identify and structurally characterise reaction components, we performed LC-DAD-SPENMR analysis. Two different approaches were used. In the first one, we filtered the reaction mixture and dissolved the crude products III and IV together with reactants in acetonitrile and injected $25 \mu \mathrm{L}$ of the solution into the LC-SPE system. The LC-DAD chromatogram of this standard mixture is presented in Figure $2 \mathrm{a}$. In the second approach, we took aliquots directly from the reaction mixture $(20 \mu \mathrm{L})$, added $480 \mu \mathrm{L}$ of acetonitrile and then performed the analysis by injecting $25 \mu \mathrm{L}$ of the solution. The chromatogram is shown in Figure $2 b$.
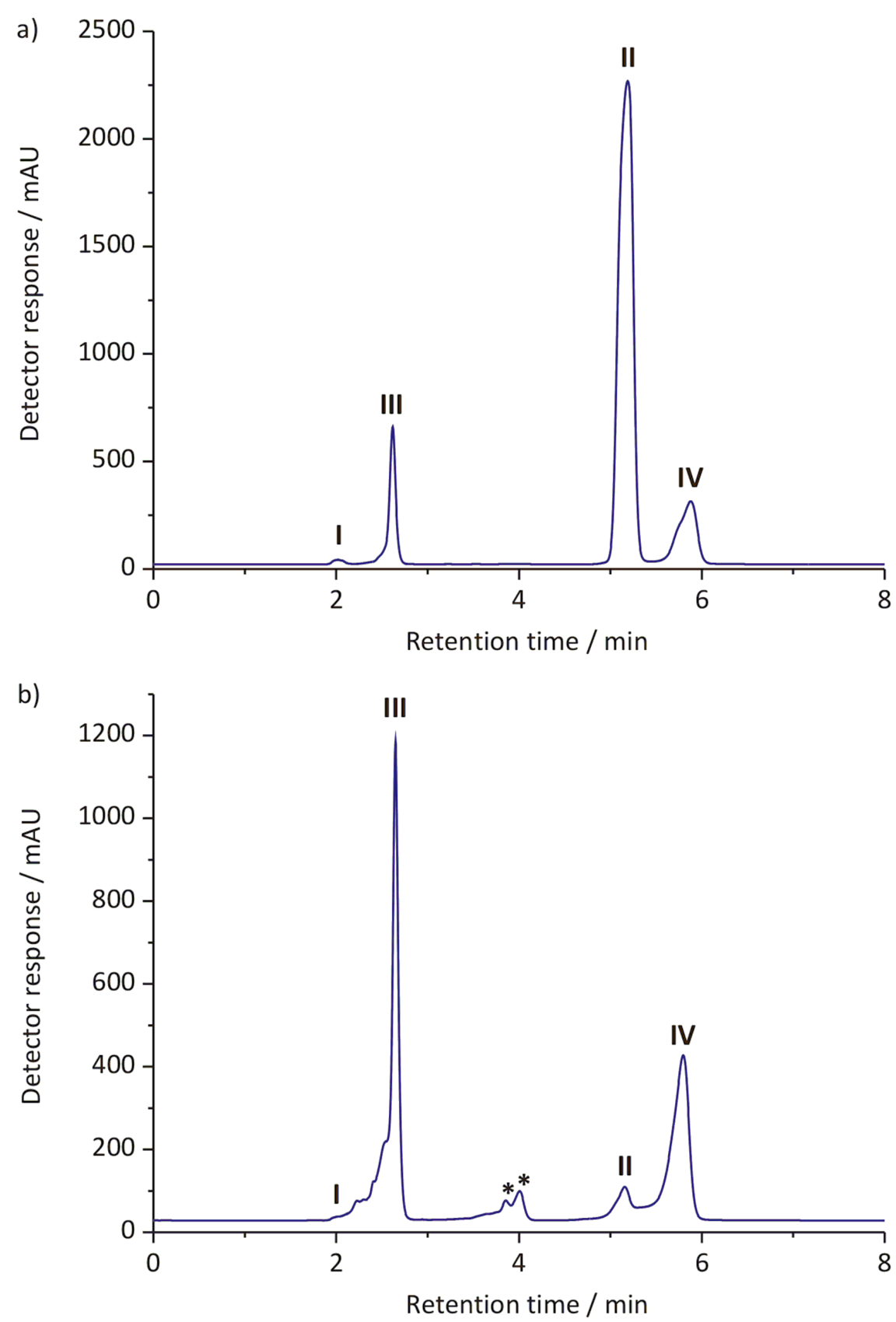

Figure 2. LC-DAD chromatogram of (a) the standard reaction mixture, (b) the reaction mixture aliquot collected $5 \mathrm{~min}$ after the reaction start. The peaks designated with asterisks are unknown compounds.

Aliquots were collected at the beginning of the reaction and after 3, 5, 10, 25 and 40 minutes. It is seen in the Figure 2 that two products were formed, III and IV with the retention times of 2.6 and 5.9 minutes, respectively. In chromatograms collected during the reaction course those peaks gradually increased while I and II decreased and vanished in the interval between 5 and 10 minutes thus indicating the end of the 
reaction. This finding is somewhat different from that previously observed by in-line vibrational spectroscopy and statistical methods [11] where the reaction endpoint in methanol was estimated to be around 30 minutes. According to LC area peak integrals condensation reactions gave $48 \%$ of III and $52 \%$ of IV which was similar to previous results. A close inspection of chromatogram depicted in Figure $2 \mathrm{~b}$ reveals two additional peaks with retention times around 4 minutes. Unfortunately, those couldn't be trapped on the cartridges used in this study and further method development is needed to resolve their identity and structure.

To identify and structurally characterise the reaction products III and IV, the contents of their LC peaks were sent to post column SPE trapping for pre-concentration prior to NMR analysis in order to achieve a better signal-to-noise ratio. Subsequently, the SPE cartridges were dried with nitrogen gas before peak elution. Deuterated acetonitrile was used to elute and transfer the peaks from the cartridges to $3 \mathrm{~mm}$ NMR tubes. To record one-dimensional proton and two-dimensional COSY spectra, a single trapping was sufficient for the compound IV (Figures 3 and 4), while multiple trapping option was used to record heteronuclear two-dimensional spectra (HSQC and HMBC).

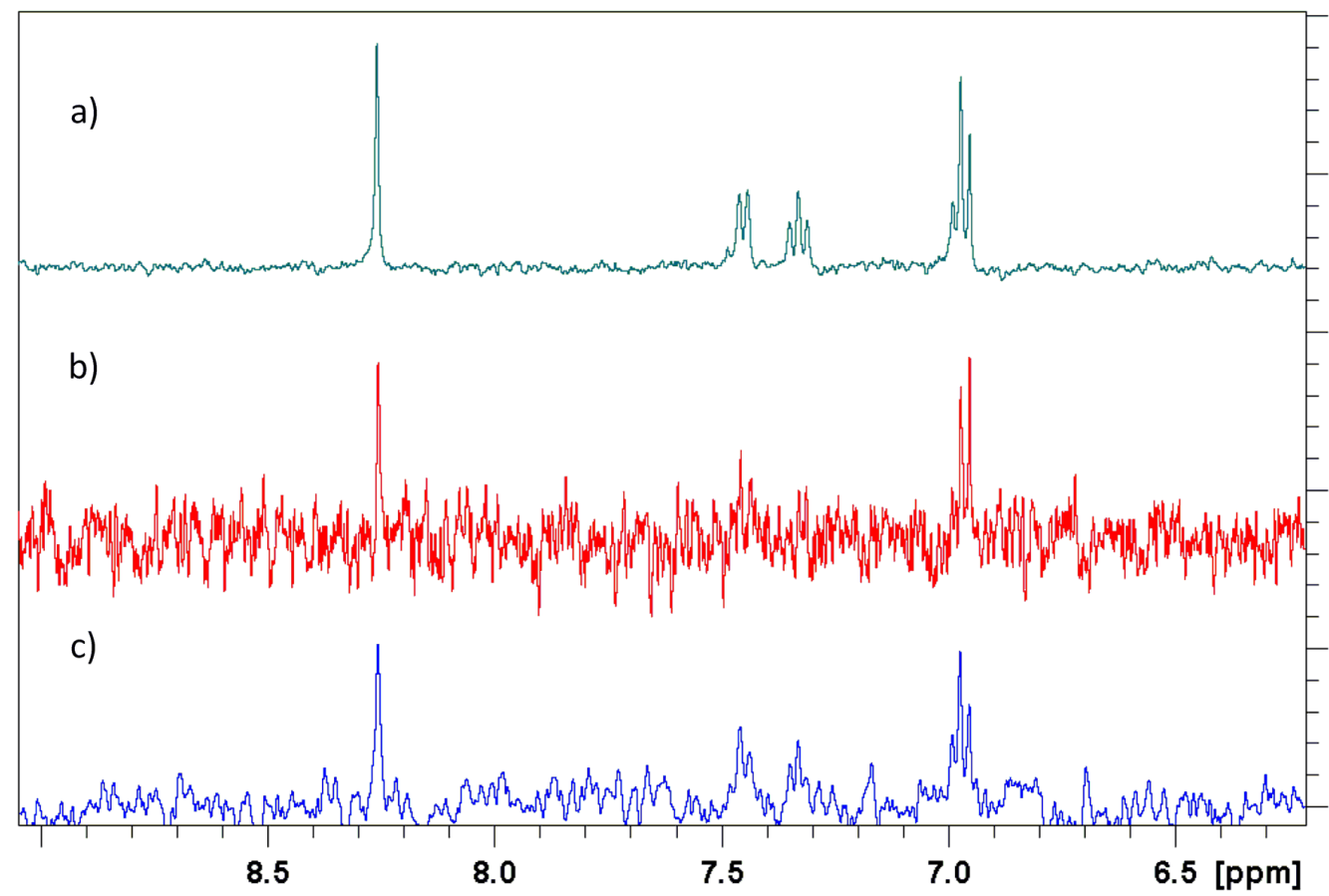

Figure 3. An expanded region of ${ }^{1} \mathrm{H}$ NMR spectrum of IV obtained in LC-SPE-NMR mode for: (a) standard reaction mixture (initial mass $1.95 \mu \mathrm{g}, 64$ scans, $6 \mathrm{~min}$ ) (b) reaction mixture aliquot collected after 5 min (64 scans, $6 \mathrm{~min}$ ) and (c) (128 scans, $12 \mathrm{~min})$.

It is noteworthy to say that the initial amount of the compound IV to record the proton spectrum displayed in Figure 3a was only $1.95 \mu \mathrm{g}$. The concentration of IV was even lower in the samples collected directly from the reaction mixture and satisfactory proton spectrum was obtained with 128 scans (Figure $3 c)$.

Furthermore, very good signal-to-noise ratio was observed also in the COSY spectrum of IV depicted in Figure 4 with the initial amount of only $24 \mu \mathrm{g}$. This clearly demonstrates the advantage of using LC-SPENMR setup with Prodigy cryoprobe for detection and identification of low concentrated samples. The structure of the compounds was unambiguously confirmed by the analysis of the recorded one- $\left({ }^{1} \mathrm{H}\right)$ and two-dimensional NMR spectra (COSY, HSQC and HMBC). 


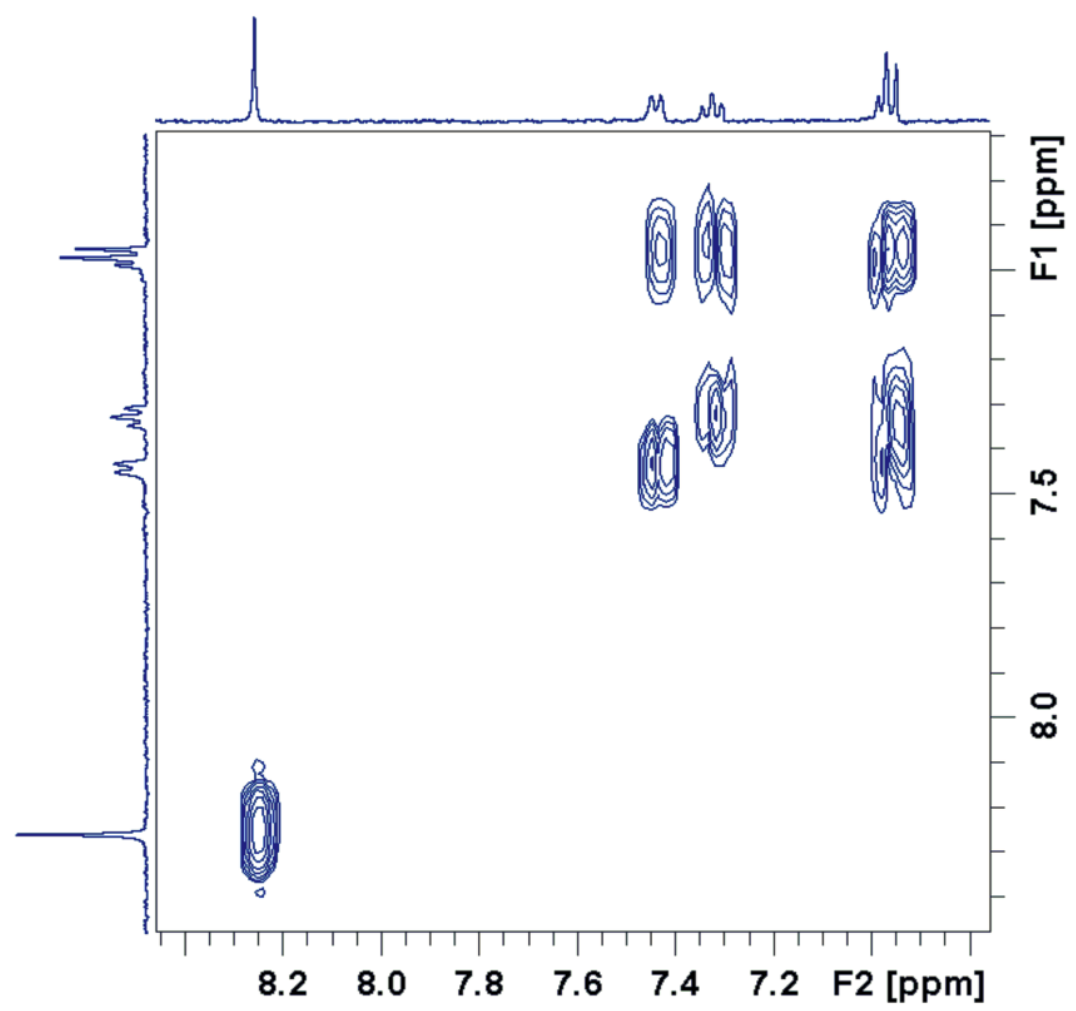

Figure 4. COSY NMR spectrum obtained in LC-SPE-NMR mode for IV isolated from the reaction mixture (16 scans, $1 \mathrm{~h}$ and $16 \mathrm{~min})$.

\section{Conclusions}

Condensation reaction of carbohydrazide with salicylaldehyde was successfully monitored by LC-DADSPE-NMR with Prodigy cryoprobe. Application of this method made it possible to identify and structurally characterise reaction products in a fast and efficient manner. After the reaction mixture components were separated on the chromatographic column and trapped on SPE cartridges, they were sent to NMR. Oneand two-dimensional NMR spectra were recorded and analysed. Spectral analysis confirmed the structures of the reaction products, e.g. mono- (III) and bis(salicylidene)carbohydrazide (IV).

\section{References}

[1] S. Barman, L. You, R. Chen, V. Codrea, G. Kago, R. Edupuganti, J. Robertus, R.M. Krug, E. V. Anslyn, European Journal of Medicinal Chemistry 71 (2014) 81-90.

[2] V.N. Telvekar, A. Belubbi, V.K. Bairwa, K. Satardekar, Bioorganic \& Medicinal Chemistry Letters 22 (2012) 2343-2346.

[3] T. Aboul-Fadl, F.A.S. Bin-Jubair, O. Aboul-Wafa, European Journal of Medicinal Chemistry 45 (2010) 4578-4586.

[4] J. Camacho, A. Barazarte, N. Gamboa, J. Rodrigues, R. Rojas, A. Vaisberg, R. Gilman, J. Charris, Bioorganic \& Medicinal Chemistry 19 (2011) 2023-2029.

[5] A.H. Corvin, J.D. Reinheimer, Journal of American Chemical Society 73(3) (1951) 1184-1186.

[6] B.F. Abdel-Wahab, A.-A. S. El-Ahl, Phosphorus, Sulfur, and Silicon, 185 (2010) 249-260.

[7] M. Sutradhar, T. R. Barman, E. Rentschler, Inorganic Chemistry Communications 39 (2014) 140-143.

[8] R. N. Patel, Inorganica Chimica Acta 363 (2010) 3838-3846.

[9] P. Novak, T. Jednačak, J. Parlov Vuković, K. Zangger, M. Rubčić, N. Galić, T. Hrenar, Croatica Chemica Acta 85 (4) (2012) 451-456. 
[10] M. Rubčić, N. Galić, I. Halasz, T. Jednačak, N. Judaš, J. Plavec, P. Šket, P. Novak, Crystal Growth \& Design 14 (2014) 2900-2912.

[11] T. Jednačak, P. Novak, A. Hodzic, O. Scheibelhofer, J. G. Khinast, J. Plavec, P. Šket, J. Parlov Vuković, Acta Chimica Slovenica 61 (2014) 161-169.

[12] S. Görög, Trends in Analytical Chemistry 69 (2015) 114-122.

[13] P. Novak, M. Cindrić, P. Tepeš, S. Dragojević, M. Ilijaš, K. Mihaljević, Journal of Separation Science 28 (2005) 1442-1447.

[14] S. Singh, T. Handa, M. Narayanam, A. Sahu, M. Junwal, R. P. Shah, Journal of Pharmaceutical and Biomedical Anaysis 69 (2012) 148-173.

[15] P. Novak, P. Tepeš, M. Cindrić, M. Ilijaš, S. Dragojević, K. Mihaljević, Journal of Chromatography A 1033 (2004), 299-303.

[16] P. Novak, P. Tepeš, M. Ilijaš, I. Fistrić, I. Bratoš, A. Avdagić, V. Gabelica Marković, M. Dumić, Journal of Pharmaceutical and Biomedical Anaysis 50 (2009) 68-72.

[17] G. Schlotterbeck, S.M. Ceccarelli, Bioanalysis 1 (2009) 549-559.

[18] S. Sturm, C. Seger, Journal of Chromatography A 1259 (2012) 50-61.

[19] J. W. Jaroszewski, Planta Medica 71 (2005) 795-802.

[20] S. Schmidt, C. Piechotta, M. Godejohann, I. Nihels, C. Mügge, Talanta, 82 (2010) 143-150.

[21] S. Christophoridou, P. Dais, L. H. Tseng, M. Spraul, Journal of Agriultural and Food Chemistry 53 (2005) 4667-4679.

[22] C. Seger, M. Godejohann, M. Spraul, H. Stuppner, F. Hadacek, Journal of Chromatography A 1136 (2006) 82-88.

[23] J. Larsen, D. Staerk, C. Cornett, S. H. Hansen, J. W. Jaroszewski, Journal of Pharmaceutical and Biomedical Anaysis 49 (2009) 839-842. 